\title{
Análise do desempenho motor em tarefas de "timing" antecipatório em idosos praticantes de esportes de interceptação
}

CDD. 20.ed. 152.3

796.018
Francys Paula CANTIERI* Inara MARQUES*
*Centro Universitário Fladélfia.

*Centro de Educação Física e Esporte, Universidade Estadual de Londrina.

\section{Resumo}

0 objetivo do presente estudo foi analisar o efeito da prática de esporte de interceptação no desempenho em uma tarefa complexa de "timing" antecipatório em idosos. Participaram do estudo 73 indivíduos, distribuídos em três grupos: idosos atletas, idosos não atletas e adultos jovens. A tarefa consistiu na execução de uma sequência de toques em quatro sensores, realizados simultaneamente ao deslocamento do estímulo, de modo que o último toque coincidisse com o acendimento do último diodo. Foram utilizadas três velocidades de acendimento dos diodos $(1 \mathrm{~m} / \mathrm{s}, 1,5 \mathrm{~m} / \mathrm{s}, 2,2 \mathrm{~m} / \mathrm{s})$, sendo realizadas 10 tentativas em cada velocidade. 0 desempenho foi analisado por meio do cálculo do erro constante, erro variável e erro absoluto. Os resultados indicaram efeito positivo da prática de esporte sobre o desempenho do "timing" antecipatório em idosos atletas. Houve também, efeito da variação da velocidade do estímulo, com os melhores desempenhos dos grupos na velocidade moderada.

UnItermos: Idosos; "Timing" antecipatório; Prática de esporte; Desempenho motor.

\section{Introdução}

O ser humano compõe um sistema capaz de identificar, processar e armazenar grandes quantidades de informaçôes provenientes do ambiente que combinadas, determinam a velocidade com que as respostas motoras são elaboradas. No entanto, o desempenho na capacidade de elaboração dessas respostas motoras apresenta uma grande variabilidade ao longo do tempo, apresentando declínios evidentes durante o processo de envelhecimento (MAGill, 2002).

Segundo BirRen (1964), a lentidão na resposta motora apresentada pelos idosos é consequência da debilidade das funções centrais e periféricas, mais especificamente, do Sistema Nervoso Central e dos mecanismos sensório-motores, que levam à diminuição da velocidade com que o idoso inicia, executa e finaliza seus movimentos (BunCE, 2001; POULTON, 1957).

Outro aspecto importante relacionado ao desempenho motor de idosos é a diminuição da capacidade de coincidir ações motoras com o deslocamento de estímulos externos, que se reflete na execução de inúmeras habilidades cotidianas como dirigir, subir em escadas rolantes e entrar em portas giratórias, denominada de "timing" antecipatório (Poulton,
1957). Essas habilidades exigem que o executante seja capaz de organizar e sincronizar suas açôes aos eventos que ocorrem no ambiente (SCHMIDT, 1969). As alterações ocorridas nas execuções das habilidades motoras em tarefas antecipatórias aumentam com o passar dos anos, contudo Cerella (1985) ressalta que, apesar de se observar diminuiçóes na velocidade de execução dos movimentos ao longo do tempo, estas alterações não têm uma relação linear com o processo de envelhecimento, devido a grande variabilidade de desempenho do "timing" antecipatório observada entre os idosos, em função da influência de fatores degenerativos, ambientais ou pelo estilo de vida adotado. Exemplo disso é a inatividade física, o desuso e as mudanças de estratégias adotadas na solução de problemas (MeEuwsen, Goode \& GogGin, 1997).

É correto afirmar, portanto, que a idade representa um papel significativo nas mudanças referentes ao desempenho do "timing" antecipatório, pois com o passar do tempo, há uma diminuição gradativa do desempenho de tarefas coincidentes, com um declínio significativo a partir dos 70 anos (BENGUIGUI, BRODERICK $\&$ Ripoll, 2004; MeEuWSen, Goode \& GogGin, 1997). 
Outra variável que pode influenciar o desempenho do "timing" antecipatório é a velocidade de deslocamento do estímulo, visto que para a obtenção de níveis satisfatórios de "timing" antecipatório é necessário que o indivíduo faça o reconhecimento correto da velocidade do estímulo, a fim de prever corretamente o momento exato da chegada do mesmo ao alvo. O estudo conduzido por PINHeIro e Corrêa (2005) indicou que a desaceleração do estímulo interferiu no desempenho do "timing" antecipatório em indivíduos de diferentes faixas etárias, mas os autores ressaltaram que os piores desempenhos foram apresentados pelos idosos. Por outro lado, o estudo de Fleury e BARDY (1985) reconheceu que os piores desempenhos no "timing" antecipatório foram verificados nas velocidades extremas, muito altas ou muito baixas. Esses autores ressaltaram que não foram capazes de identificar uma relação direta entre o desempenho do "timing" e o aumento da velocidade do estímulo, no entanto, afirmaram que parece haver uma velocidade intermediária considerada ideal, porém ainda indeterminada, que propicia os melhores desempenhos.

Atualmente, há a premissa de que a prática de exercícios físicos pode desacelerar as alterações advindas do envelhecimento e, até mesmo, prevenir tais perdas (Christensen, Payne, Wughalter, Yan, Henehan \& JONES, 2003). Assim, tem sido cada vez mais difundida a ideia de que a prática sistemática de atividade física pode influenciar positivamente no sistema funcional e nos níveis de capacidades motoras relacionadas à saúde, tais como força, flexibilidade e coordenação motora (Guimaräes \& Farinatti, 2005; Krause, BuZzachera, Hallage, Pulner \& Silva, 2007; Mazzeo, Cavanagh, Evans, Fiatarone, Hagberg, McAuley \& Startzell, 1998; Nunes \& SANTOS, 2009), assim como, atuar em açôes preventivas de doenças como diabetes, hipertensão e obesidade, reduzindo a mortalidade e, por consequência, promovendo a melhora física, social e emocional desses indivíduos (SPIRDUSO, 2005).

\section{Métodos}

\section{Participantes}

Participaram desse estudo 73 indivíduos destros do sexo masculino, dos quais 49 compuseram o grupo de idosos (idade entre 60 e 90 anos) e 24 compuseram o grupo de adultos jovens (idade entre 20 a 30 anos). Os indivíduos foram divididos em três grupos: $\mathrm{G} 1(\mathrm{~N}=22)$ composto por tenistas,
Alguns estudos têm apontado para o efeito positivo da prática de atividades físicas sistemáticas no desempenho do "timing" antecipatório, propiciando aos indivíduos possibilidades de desenvolver formas eficazes de realizar ações coincidentes a partir de experiências adquiridas, podendo alcançar índices de desempenho bastante satisfatórios (ABOUREZK \& Toole, 1995; Christensen et AL., 2003; Colcombe \& Kramer, 2003; Ericsson, Krampe \& TeschRÖMER; 1993; Krampe \& ERICSSON, 1996; Santos, 2005; TeIXeIra, 2006). Assim, pode-se supor que, a prática regular de esporte de interceptação pode, igualmente, interferir no desempenho do "timing" antecipatório em indivíduos que se encontraram em processo de envelhecimento (BENGUIGUI \& RipOlL, 1998; Lobjois, Benguigui \& Bertsch, 2006).

Diante dessas informações, destaca-se a importância do estudo do "timing" antecipatório, por se tratar de um componente importante da velocidade de resposta do indivíduo, exigido na execução de várias atividades cotidianas, que sofre declínios evidentes durante o processo de envelhecimento. Além disso, ainda não há resultados acerca do efeito da prática de esporte de interceptação, sobre a capacidade de "timing" antecipatório, que pode apresentar diferenças significativas no desempenho em função das variáveis envolvidas na tarefa. Ademais, não há resultados que indiquem a prevalência de alguma modalidade específica com resultados favoráveis no desempenho do "timing" antecipatório, tampouco na prática de tarefas de interceptação, ou seja, aqueles que exijam a coincidência entre a ação motora e a chegada de um estímulo externo, como por exemplo, o tênis de campo.

Com isso, esse estudo teve como objetivo analisar o desempenho motor em tarefas de "timing" antecipatório com variação de velocidade de estímulo, em indivíduos idosos praticantes de esporte de interceptação. praticantes regulares, com idade média de 69 anos, G2 $(\mathrm{N}=27)$ composto por indivíduos não praticantes de esportes de interceptação e não praticantes de atividades físicas regulares, com idade média de 68,3 anos e G3 $(\mathrm{N}=24)$ composto por indivíduos não atletas com idade média de 23,5 anos. Cabe ressaltar que não foi controlada a prática de outras atividades recreativas dos participantes. 
Todos os participantes foram considerados completamente independentes, segundo instrumento de avaliação das atividades da vida diária proposto por LAWTON e BRody (1969). Os procedimentos adotados na pesquisa obedeceram aos Critérios de Ética em pesquisa com seres humanos do Comitê de Ética da Universidade Local, sob o parecer $n^{\circ}$ 119/08, conforme resolução no 196/96 do Conselho Nacional de Saúde.

TABELA 1 - Número de integrantes e idade média dos grupos.

\begin{tabular}{lccc}
\hline Grupos & N & Idade média & SD \\
\hline G1- Atletas & 22 & 69 & 8,8 \\
G2- Não Atletas & 27 & 68,3 & 8,3 \\
G3- Adultos & 24 & 23,5 & 3,2 \\
\hline
\end{tabular}

\section{Descrição do equipamento}

O instrumento utilizado foi o aparelho de "timing" coincidente em tarefas complexas e patenteado sob o número P.I. 0.403.433-4 (CORRÊA \& TANI, 2004). Esse aparelho é composto por uma canaleta de 195 centímetros de comprimento, oito centímetros de largura e dois centímetros de altura sobre a qual estão dispostos 96 diodos com distância de um centímetro e meio entre eles. Para que os participantes tivessem uma completa visualização do equipamento, a parte final da canaleta ficou apoiada sobre uma mesa de 70 centímetros de comprimento, 70 de largura e 74 de altura e a sua parte inicial ficou sustentada por um suporte afixado na parede, com uma inclinação vertical de 45 graus. Nesta mesa, além da parte final da canaleta, ficavam dispostos quatro sensores (medindo cinco centímetros de largura e 15 centímetros de comprimento), posicionados conforme uma ordem de toques pré-estabelecida, e um computador contendo um "software", desenvolvido especificamente para este estudo (CANTIERI, CANTIERI \& MARQUeS, 2008) que possibilitava a variação da velocidade de deslocamento do estímulo, além de indicar o tempo decorrido entre a apresentação do estímulo e a execução da resposta (FIGURA 1).

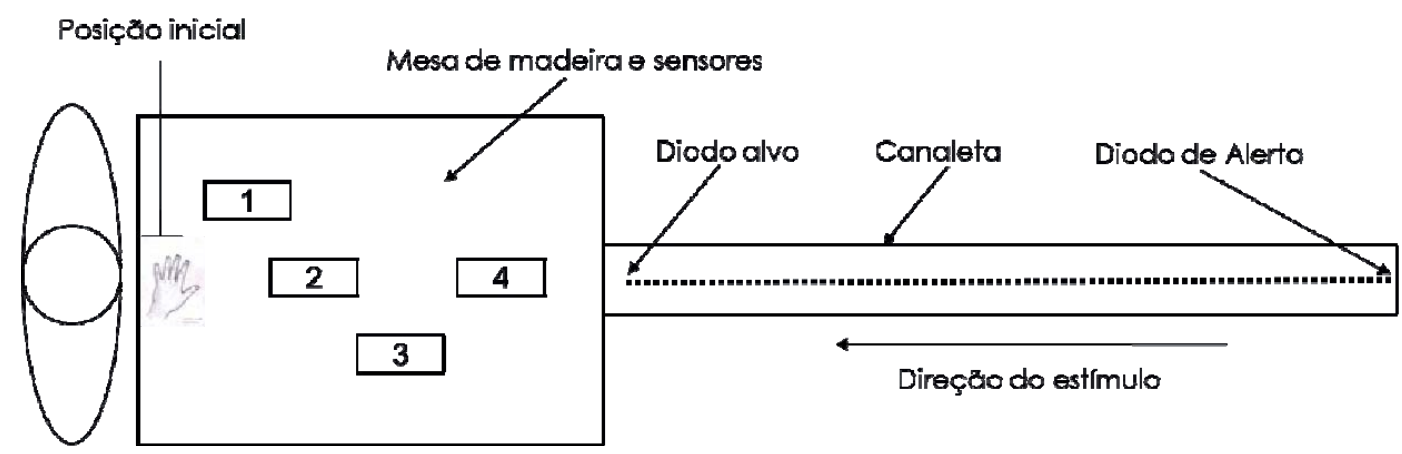

FIGURA 1 - Ilustração esquemática do aparelho de "timing" coincidente em tarefas complexas, adaptado de PAROLI e TANI (2009).

\section{Procedimentos}

A execução da tarefa foi realizada individualmente e o participante foi posicionado sentado em frente ao aparelho, de modo que ele pudesse visualizar o deslocamento do estímulo, apresentado pelo acendimento de todos os diodos. Os participantes calçaram uma luva de alumínio em sua mão dominante (todos os indivíduos eram destros) e a posicionaram sobre um alvo que determinava o inicio da tarefa. A tarefa consistiu em realizar, após o sinal de alerta (diodo 1 ), uma sequência de quatro toques na ordem 1-2-3-4, em três velocidades diferentes (velocidade lenta de 1 $\mathrm{m} / \mathrm{s}$, velocidade moderada de $1,5 \mathrm{~m} / \mathrm{s}$ e velocidade rápida de $2,2 \mathrm{~m} / \mathrm{s}$ ). Os quatro toques deveriam ser realizados simultaneamente ao deslocamento do estímulo, de modo que o último toque (sensor 4) coincidisse com o acendimento do último diodo da canaleta, que representava a chegada do estímulo (diodo alvo). Foram realizadas cinco tentativas da tarefa para familiarização e 10 tentativas em cada velocidade, apresentadas aleatoriamente, totalizando 30 tentativas.

\section{Medidas de desempenho}

Foram utilizadas como medidas de desempenho o erro constante (EC) representando a tendência direcional do erro, o erro absoluto (EA) representando a precisão com que a meta foi atingida, e o erro variável (EV) representando a consistência do erro (SCHMidT \& LEE, 1999). 


\section{Análise estatística}

$\mathrm{Na}$ análise descritiva foi adotada a mediana como medida de tendência central.

Devido aos dados analisados não terem apresentado normalidade, foi adotado o teste de
Kruskall-Wallis para verificar a diferença entre os grupos, com "post hoc" U de Mann Whitney. O teste de Friedman foi utilizado para verificar as diferenças entre as velocidades. $\mathrm{O}$ nível de significância adotado foi de $p<0,05$. O programa estatístico utilizado foi o SPSS, versão 13.0.

\section{Resultados}

Os resultados referentes à análise intergrupos indicaram que houve diferenças no desempenho entre os três grupos, nas diferentes velocidades.

$\mathrm{Na}$ velocidade 1, houve diferença entre os grupos G1, G2 e G3 apenas para o erro constante (H (2, $30)=7,13 ; \mathrm{p}<0,05)$ e para o erro absoluto $(\mathrm{H}$ $(2,30)=7,76, p<0,05)$. Sendo essas diferenças encontradas no erro constante, entre o grupo G1 e G2 $(Z=-2,28 ; p<0,05)$ e $G 2$ e G3 $(Z=-2,23$; $\mathrm{p}<0,05)$. No erro absoluto entre o grupo G2 e $\mathrm{G} 3(\mathrm{Z}=-2,73 ; \mathrm{p}<0,01)$. Observa-se que não foram encontradas diferenças significativas entre os grupos G1 e G3 em nenhum dos erros analisados (FIGURA 2).

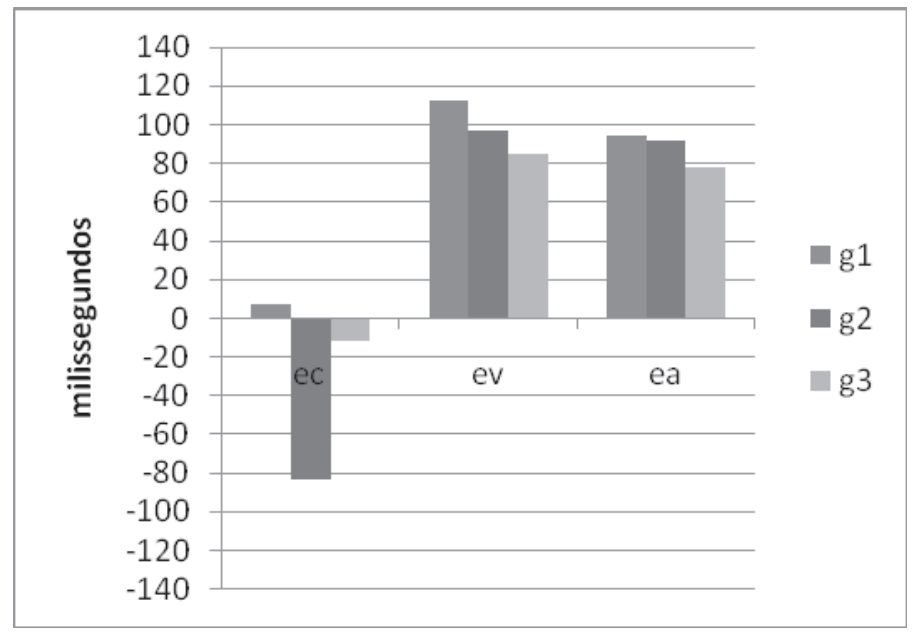

FIGURA 2 - Mediana do erro constante, erro variável e erro absoluto (milissegundos) dos grupos G1, G2 e G3, na velocidade lenta $(1 \mathrm{~m} / \mathrm{s})$.

$\mathrm{Na}$ velocidade 2, houve diferenças significativas entre os grupos G1, G2 e G3 para o erro constante $(\mathrm{H}(2,30)=19,88 ; \mathrm{p}<, 0,01)$, para o erro variável $(\mathrm{H}(2,30)=9,13 ; \mathrm{p}=0,01)$ e para o erro absoluto $(\mathrm{H}(2,30)=6,34 ; \mathrm{p}<0,05)$. O teste U de MannWhitney localizou essas diferenças entre o grupo
G1 e G2 no erro constante $(Z=-3,46 ; p<0,05)$, entre os grupos $\mathrm{G} 1$ e $\mathrm{G} 3$ no erro variável $(Z=-2,91$; $\mathrm{p}<0,01)$ e erro absoluto $(Z=-2,40 ; p=0,01)$, e entre os grupos $\mathrm{G} 2$ e $\mathrm{G} 3$ no erro constante $(\mathrm{Z}=$ $-4,09 ; \mathrm{p}<0,01)$ e erro variável $(Z=-2,03 ; p<0,05)$ (FIGURA 3). 


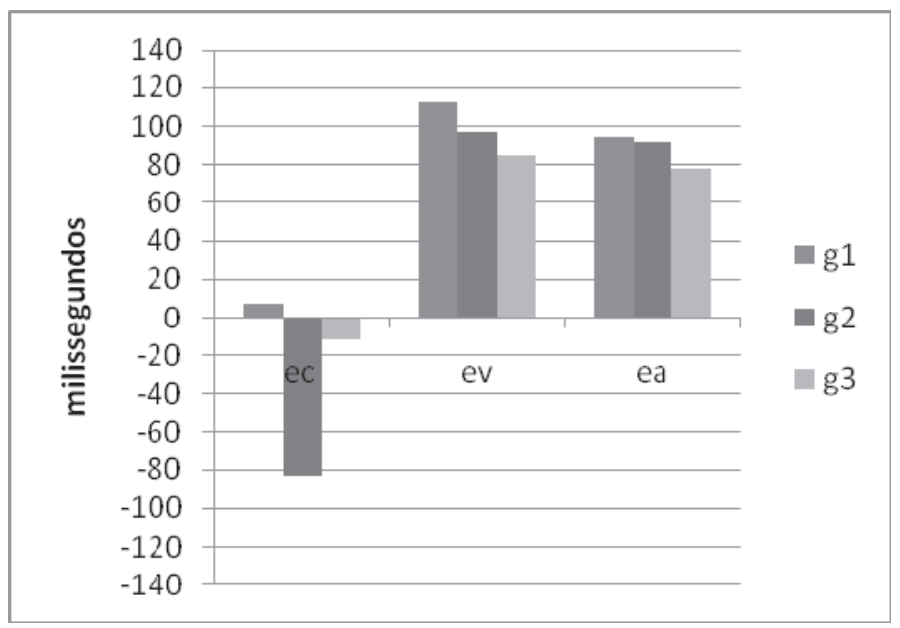

FIGURA 3 - Mediana do erro constante, erro variável e erro absoluto (milissegundos) dos grupos G1, G2 e G3, na velocidade moderada $(1,5 \mathrm{~m} / \mathrm{s})$.

$\mathrm{Na}$ velocidade 3 , houve diferenças significativas entre os grupos G1, G2 e G3 para o erro constante $(\mathrm{H}(2,30)=29,64 ; \mathrm{p}<0,01)$, erro variável $(\mathrm{H}(2,30)$ $=26,59 ; \mathrm{p}<0,01)$ e para o erro absoluto $(\mathrm{H}(2,30)$ $=30,62 ; \mathrm{p}<0,01)$. O teste U de Mann- Whitney localizou essas diferenças entre o grupo G1 e G2 no erro constante $(Z=-3,76 ; p<0,01)$, no erro variável $(Z=-3,67 ; p<0,01)$ e no erro absoluto $(Z$ $=-3,73 ; p<0,01)$. Entre os grupos G1 e G3 no erro constante $(Z=-1,98 ; p<0,05)$ e erro absoluto $(Z=$ $-2,41 ; p=0,01)$. Entre os grupos G2 e G3 no erro constante $(Z=-5,01 ; p<0,01)$ e no erro variável $(Z=-4,81 ; p<0,01)$ e erro absoluto $(Z=-5,03 ; p$ $<0,01)$ (FIGURA 4).

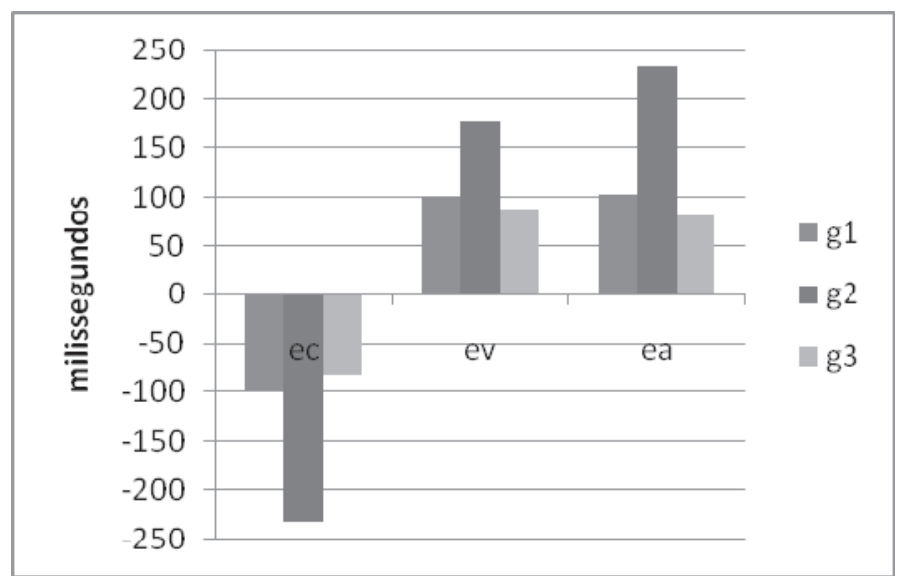

FIGURA 4 - Mediana do erro constante, erro variável e erro absoluto (milissegundos) dos grupos G1, G2 e G3, na velocidade rápida $(2,2 \mathrm{~m} / \mathrm{s})$.

A análise intragrupo indica que houve um efeito da variação da velocidade do estímulo em todos os grupos analisados.

Com relação ao grupo G1, o teste de Friedman apontou diferenças significativas entre as velocidades apenas para o erro constante $\mathrm{X}^{2}[(\mathrm{df}=2, \mathrm{n}=22)=18,09] \mathrm{e} \mathrm{p}<$ 0,05 , estando essas diferenças localizadas entre as velocidades 1 e 2 e entre 2 e $3(Z=-3,13 ; p<0,01)$, sendo o maior atraso em ambos os casos observado na velocidade 3 .

Para o grupo G2, observou-se diferenças significativas entre as velocidades para o erro constante $\mathrm{X}^{2}[(\mathrm{df}$
$=2, \mathrm{n}=27)]=27,18$ e $\mathrm{p}<0,05$, para o erro variável $\mathrm{X}^{2}[(\mathrm{df}=2, \mathrm{n}=27)]=14,74$ e $\mathrm{p}<0,05$, para o erro absoluto $\mathrm{X}^{2}[(\mathrm{df}=2, \mathrm{n}=27)]=26,63$ e $\mathrm{p}<0,05$.

Quanto ao erro constante houve diferenças entre a velocidade 1 e $3(Z=-4,13$; $p<0,01)$ e 2 e $3(Z=-4,18$; $p<$ $0,01)$, sendo os maiores atrasos observados na velocidade 3 .

Quanto ao erro variável houve diferenças entre as velocidades 1 e $2(Z=-2,64 ; p<0,01)$, entre as velocidades 1 e $3(Z=-2,04 ; p<0,05)$ e entre as velocidades 2 e $3(Z=-3,36 ; p<0,01)$, sendo o pior desempenho também observado na velocidade 3 . 
Quanto ao erro absoluto encontrou-se diferenças entre as velocidades 1 e $3(Z=-4,18 ; p<0,01)$ e velocidades 2 e $3(Z=-4,10 ; p<0,01)$ e novamente os piores desempenhos foram observados na velocidade 3 .

Quanto ao grupo G3, houve diferenças significativas entre as velocidades apenas para o erro constante $X^{2}[(d f=2, n=24)]=15,25$ e p < 0,05 , estando essas diferenças entre as velocidades 1 e $2(Z=-3,0 ; p<0,01), 1$ e $3(Z=-2,2 ; p$ $<0,05)$ e 2 e $3(Z=-3,74 ; p<0,01)$, sendo os piores desempenhos verificados na velocidade 3 (TABELA 2).

TABELA 2 - Mediana (milissegundos) dos erros constante (EC), variável (EV) e absoluto (EA), dos grupos G1, G2 e G3, nas três velocidades de estímulo (V1, V2, V3).

$\mathrm{G} 1=$ idosos atletas; G2=idosos nãoatletas; G3 = adultos.

\begin{tabular}{cccccccccc}
\hline & & G1 & & & G2 & & \multicolumn{3}{c}{ G3 } \\
\hline & V1 & V2 & V3 & V1 & V2 & V3 & V1 & V2 & V3 \\
\hline EC & $-39,6$ & 7,25 & $-99,2$ & $-97,2$ & $-83,5$ & -233 & $-56,1$ & -12 & $-81,4$ \\
EV & 132,3 & 112,4 & 100,3 & 129 & 96,9 & 178,7 & 119,9 & 84,6 & 89,9 \\
EA & 100,1 & 94,7 & 102,6 & 106,1 & 92,1 & 233 & 85,4 & 77,8 & 81,4 \\
\hline
\end{tabular}

De modo geral, o grupo 3 (jovens), apresentou melhores desempenhos nas três velocidades testadas. O grupo 1 (atletas) foi bastante semelhante ao grupo 3 na velocidade lenta, onde não foram encontradas diferenças significativas. Além disso, houve o efeito da variação da velocidade do estímulo em todos os grupos analisados, que apresentaram piores desempenhos na velocidade alta, com exceção do grupo 3 que foi mais variável e mais impreciso na velocidade 1

\section{Discussão}

Este estudo teve como objetivo analisar o desempenho motor em tarefas de "timing" antecipatório com variação de velocidade de estímulo, em indivíduos idosos praticantes de esporte de interceptação.

Os resultados dessa pesquisa apontaram para a existência de um efeito positivo da prática de esporte de interceptação sobre o desempenho do "timing" antecipatório, nos quais o grupo de idosos praticante de esporte de interceptação, no caso o tênis, obteve desempenho superior ao grupo de idosos não praticante de esporte de interceptação da mesma faixa etária.

Esse resultado encontra suporte no trabalho conduzido por Colcombe e Kramer (2003). Esses autores sugerem que há uma influência positiva da manutenção de práticas de atividades físicas sobre o desenvolvimento cognitivo do idoso, desde que se leve em consideração o tipo e o tempo de intervenção utilizada, o sexo testado, entre outros. Nesse sentido, a prática de esporte de intervenção parece ter sido um diferencial em nosso estudo, ressaltado pelos dados obtidos.

Os resultados do presente estudo, também são corroborados pelos de ABOUREZK e TOOLE (1995) que objetivaram verificar o efeito da prática de exercícios físicos sobre a velocidade de resposta motora em idosas. Os resultados apontaram para uma superioridade de desempenho das idosas ativas, sugerindo que as idosas ativas não experimentaram as reduções de inibição do sistema nervoso central advindas do envelhecimento, com a mesma magnitude que as não ativas.

Também foi encontrado suporte nos resultados obtidos no estudo conduzido por CHRISTENSEN et al. (2003) que identificou relações entre os níveis de atividade física e o desempenho de idosos ativos quando comparados aos inativos. Ressalta-se que o estudo utilizou-se de tarefas de tempo de reação simples, tempo de reação de escolha e "timing" antecipatório.

Os estudos acima mencionados parecem confirmar a hipótese de que a manutenção da prática de atividades físicas é um importante aliado no processo de envelhecimento, influenciando satisfatoriamente, na execução de tarefas que envolvem a coincidência de eventos. Tal reflexão foi compartilhada com ERicsson, Krampe e Tesch-RÖmer (1993) e Krampe e Ericsson (1996). Assim, diante dos resultados do presente estudo, pode-se supor que indivíduos que praticam esporte de interceptação, como o tênis de campo, podem manter seu desempenho em tarefas que envolvam coincidência de eventos, uma vez que são estimulados aspectos percepto-motores importantes a várias atividades motoras e cotidianas. 
O bom desempenho apresentado pelo grupo de idosos praticantes de esporte de interceptação, mesmo entre os indivíduos mais velhos, ressalta a capacidade dos mesmos em alcançar índices de desempenhos satisfatórios, solucionando problemas impostos pelo ambiente em constante alteração, mediante as estratégias adotadas por eles na execução de suas tarefas, utilizandose das experiências adquiridas ao longo da vida (LoBJoIs, Benguigui \& Bertsch, 2006; Santos, 2005).

BENGUigur e RiPOLL (1998) também ressaltaram a importância das ações interceptativas na percepção e na elaboração da resposta motora. Seus resultados mostraram uma aceleração do desenvolvimento percepto-motor em jovens praticantes de esporte, em razão da prática constante de tarefas envolvendo o "timing” antecipatório. Poder-se-ia supor, portanto, que a prática de esporte de interceptação pudesse provocar adaptações percepto-motoras, também em indivíduos que se encontram em processo de envelhecimento, mantendo essa capacidade em níveis desejáveis.

Isso, por que, em se tratando da comparação entre os atletas idosos e os adultos jovens, observou-se que ambos os grupos obtiveram desempenhos bastante semelhantes em algumas medidas de erro analisadas. Se fossem analisados, por exemplo, apenas os dados relativos à velocidade de estímulo lenta, não teriam sido encontradas diferenças significativas entre os grupos de idosos atletas e o de adultos jovens, reforçando a importância da prática de esporte na manutenção da capacidade de "timing" antecipatório. Esse resultado vai ao encontro dos achados de KRAMPE e ERICSSON (1996) que investigaram a influência da continuidade da prática de habilidades motoras na manutenção da capacidade cognitivo-motora em pianistas experientes, identificando que os pianistas experientes (60 anos) obtiveram desempenhos bastante similares aos dos adultos jovens ( 25 anos) e superiores aos inexperientes da mesma faixa de idade.

Resultados análogos foram verificados por LOBjoIs, Benguigui e BerTsCh (2006) que apontaram uma similaridade no desempenho do "timing" antecipatório, entre os idosos atletas e os jovens atletas. Os autores atribuíram as similaridades no desempenho entre os dois grupos à hipótese de compensação, a qual sugere que os idosos são capazes de compensar suas perdas pelas experiências adquiridas.

Só há uma discordância com a literatura, quanto aos resultados obtidos no presente estudo. MEEUWWEN, Goode e GogGin (1997) sugeriram haver pouco efeito da prática de esporte sobre o desempenho do "timing" antecipatório em mulheres. Eles analisaram o desempenho do "timing" antecipatório em mulheres adultas e idosas atletas com idades entre 22 e 75 anos, destacando o melhor desempenho obtido pelo grupo de adultas jovens em relação às idosas, a despeito de também serem atletas. Como os dados apresentados são sobre a população feminina, não há correspondência suficiente com os dados do presente estudo.

A semelhança dos resultados obtidos entre idosos atletas e adultos jovens, observada no presente estudo, pode ter ocorrido em função tanto da capacidade dos idosos atletas em compensar suas perdas através das experiências adquiridas, quanto pela manutenção da prática de esportes que envolvem a sincronização de movimentos, ao longo do processo de envelhecimento. Contudo essa compensação apresentada pelos idosos atletas parece estar relacionada à velocidade de deslocamento do estímulo, mostrando-se eficiente apenas nas velocidades lentas apresentadas nesse estudo.

Já, com relação às diferenças encontradas entre o grupo de idosos praticantes de esporte de interceptação e o grupo de idosos não praticantes, as explicações são congruentes com os resultados obtidos no estudo de LOBJOIs, BENGUIGUI e BERTSCH (2006). No presente estudo os idosos não praticantes apresentaram respostas mais atrasadas e foram mais susceptíveis às variações da velocidade do estímulo, assim como os resultados do trabalho acima citado.

Essa variação no desempenho em razão das diferentes velocidades do estímulo, nos diferentes grupos sugere uma interferência no desempenho quanto à variação da velocidade do estímulo, resultantes da variação da velocidade e não pelo aumento progressivo da mesma, visto que não observou-se uma queda de desempenho em função do aumento progressivo da velocidade lenta para rápida.

De maneira geral, os grupos se mostraram mais consistentes na velocidade intermediária, apresentando piores desempenhos nas velocidades extremas (baixas e altas). Esses resultados são coerentes com os resultados obtidos por FleUrY e BARDY (1985), indicando haver uma velocidade de apresentação de estímulo ideal, compatível com o tempo necessário para o processamento da informação. Os autores sugerem que em uma velocidade muito lenta, o sujeito não conseguiria retardar a efetuação do seu plano de ação, tendendo a um declínio da precisão e inconsistência da resposta, no caso, a consistência do erro. Ao contrário, caso o tempo de apresentação do estímulo fosse muito próximo ao tempo de reação (aproximadamente 200 milissegundos), o executante teria pouco tempo para processar a informação, tendendo a apresentar desempenhos mais atrasados, mais variáveis e menos precisos. Porém, deve-se ressaltar que, no presente estudo, o tempo mínimo de 
estímulo apresentado foi superior a 800 milissegundos, dando ao executante tempo suficiente para os processos internos serem efetuados.

Os resultados indicaram, também, que o grupo de idosos atletas não variou o seu desempenho de forma significativa entre as três velocidades apresentadas, ou seja, eles conseguiram se adaptar às variaçōes da velocidade do estímulo e mantiveram os seus desempenhos semelhantes entre as modificações das mesmas. Apresentando divergência aos resultados apresentados pelos idosos atletas, o grupo de idosos não atletas apresentou uma grande variabilidade mediante a alteração da velocidade do estímulo, ressaltando os piores desempenhos àqueles encontrados nas velocidades extremas.

Quanto ao grupo de adultos jovens, apesar de terem mostrado certa variabilidade conforme a velocidade se modificava, observou-se que apenas a velocidade mais baixa foi perturbadora do seu desempenho, visto que nas outras duas velocidades, o

\section{Conclusão}

De acordo com os resultados desse estudo, tem-se claro que a idade é um fator limitante do desempenho motor dos idosos.

No entanto, a prática de esporte de interceptação como, por exemplo, o tênis de campo, tem demonstrado ser uma eficiente ferramenta no que se refere à amenização dos efeitos deletérios do envelhecimento sobre o desempenho motor do idosos, principalmente na manutenção da capacidade do "timing" antecipatório, permitindo-os serem capazes de realizar suas atividades cotidianas de forma independente, alcançando assim, uma melhor qualidade de vida.

Em resumo, os resultados indicaram vantagens no desempenho dentre os indivíduos praticantes de esporte de interceptação e parece ser um bom indicativo de que essa prática ao longo da vida pode representar os mesmos ganhos, ou até maiores, que os praticantes de atividades físicas generalizadas. Essa afirmativa pode ser justificada pelo fato dos idosos praticantes de esporte de interceptação do presente estudo terem apresentado desempenhos semelhantes aos encontrados pelos adultos jovens em algumas variáveis analisadas, como velocidade lenta de estímulo, e superaram o desempenho observado entre os idosos não atletas.

A variação da velocidade de apresentação do estímulo, também, demonstrou ter tido um efeito no grupo apresentou desempenhos bastante semelhantes. Esses resultados corroboram os achados de PINHEIRO e CORRREA (2005), que apontaram maior variabilidade entre os idosos e maior consistência entre os adultos mediante a alteração da velocidade do estímulo.

$\mathrm{E}$, por último, quanto aos resultados que refletem a precisão com que os indivíduos atingiram o alvo observou-se que tanto o grupo de idosos praticantes de esporte de interceptação quanto o grupo de adultos jovens não apresentaram diferenças no desempenho entre as três velocidades apresentadas. Segundo FLEURY e BARD (1985), os adultos jovens apresentam uma capacidade de integrar os mecanismos perceptivo e efetor, se adaptando as variaçoos da velocidade do estímulo. Como não houve diferença no desempenho dos idosos praticantes de esporte de interceptação, pode-se supor que tenha havido a mesma integração observada entre os adultos jovens, destacando o efeito da prática de esporte no desempenho dos idosos atletas.

desempenho em tarefas de "timing" antecipatório, em todos os grupos analisados, destacando, porém, que as variabilidades no desempenho não ocorreram pelo aumento da velocidade em si, mas pelas modificaçóes ocorridas entre um bloco de tentativas e outro. Ressalta-se, porém, que a variabilidade no desempenho relativo à mudança na velocidade do estímulo parece ter afetado mais àqueles indivíduos que não praticaram esporte de interceptação, fator que reforça a manutenção da prática ao longo da vida.

A partir desses resultados, caracterizar um indivíduo como idoso vai além dos aspectos fisiológicos, ou seja, deve ser considerado também, as adaptações promovidas nesse organismo envelhecido e a influência das experiências adquiridas pelos idosos ao longo do tempo que, somadas, podem alterar a velocidade com que cada indivíduo experimenta o envelhecimento. Deve-se destacar a importância da realização desse estudo do ponto de vista acadêmico e aplicado, pela sua contribuição à compreensão dos processos relacionados ao envelhecimento e por apresentar resultados que direcionam aos possíveis benefícios $\mathrm{da}$ manutenção da prática de esporte ao longo da vida, sobre o desempenho motor dos idosos. Sugere-se em estudos futuros o controle de variáveis como aceleração e desaceleração do estímulo, assim como o efeito da prática de outros esportes de interceptação, sobre o desempenho do "timing" antecipatório. 


\begin{abstract}
Analysis of motor performance in timing anticipatory tasks in elderly practioners of interception sports

The aim of this study was to analyze the effect of interception sports on the performance in of a complex anticipatory timing task in the elderly. The study included 73 participants divided into three groups: elderly athletes, elderly and young non-athletes. The task consisted in the execution of a sequence of touchs in four sensors, simultaneously to the lighting of diodes aligned in a groove, with the last touch matching the lighting of the last diode. We used three speeds of diode lighting $(1 \mathrm{~m} / \mathrm{s}, 1.5 \mathrm{~m} / \mathrm{s}, 2.2 \mathrm{~m} / \mathrm{s})$, with ten trials for each speed. The performance was analyzed by calculating the constant error, variable error and absolute error. The results indicated positive effect of sports on the anticipatory timing performance in elderly athletes. There was also the effect of varying the speed of the stimulus, with the best performances of the groups at moderate speed.
\end{abstract}

UNITERMS: Elderly; Anticipatory timing; Sports practice; Motor performance.

\title{
Resumen
}

Análisis del desempeño motor en las tareas de "timing" de previsión en ancianos practicantes de deporte de interceptación

Análisis del desempeño motor en las tareas de "timing" de previsión en ancianos practicantes de deporte de interceptación El objetivo de este estudio fue analizar el efecto de la practica de deportes de intercepción en el desempeño en una tarea compleja de "timing" de previsión en ancianos. Participaron de este estudio 73 pacientes divididos en tres grupos: ancianos deportistas, ancianos no-deportistas y jóvenes. La tarea consistió en la ejecución de una secuencia de toques en cuatro sensores, realizados simultáneamente con el desplazamiento del estímulo, de modo que el último toque coincidiese en con el encendimiento del último diodo. Se utilizaron tres velocidades de encendimiento de los diodos $(1 \mathrm{~m} / \mathrm{s} \mathrm{1,5} \mathrm{m/s} \mathrm{2,2} \mathrm{m/s),} \mathrm{siendo}$ efectuados diez intentos en cada velocidad. El rendimiento fue analizada mediante el cálculo de la constante de error, del error variable y del error absoluto. Los resultados indicaron efecto positivo de la práctica deportiva en el desempeño de "timing" de previsión en los ancianos deportistas. Hubo también el efecto de variar la velocidad del estímulo, con los mejores rendimientos de los grupos en la velocidad moderada.

Palabras clave: Ancianos; "Timing" de previsión; Práctica deportiva; Desempeño motor.

\section{Referências}

ABOUREZK, T.; TOOLE, T. Effect of task complexity on the relationship between physical fitness and reaction time in older women. Journal of Aging and Physical Activity, Champaign, v.3, p.251-60, 1995.

BENGUIGUI, N.; BRODERICK, M.; RIPOLL, H. Age differences in estimating arrival-time. Neuroscience Letters, Toronto, v.369, p.197-202, 2004.

BENGUIGUI, N.; RIPOLL, H. Effects of tennis practice on the coincidence timing accuracy of adults and children. Research Quarterly for Exercise and Sport, Washington, v.69, n.3, p.217-23, 1998.

BIRREN, J.E. The psychology of aging. Englewood Cliffs: Prentice-Hall, 1964.

BUNCE, D. The lócus of age $\mathrm{x}$ health- related physical fitness interactions in the serial choice responding as a function of task complexity: central processing or motor function? Experimental Aging Research, London, v.27, p.103-22, 2001. CANTIERI, A.R.; CANTIERI, F.P.; MARQUES, I. Software para análise de antecipação temporal em ações motoras “Timing” Rovitec, 2008. (não patenteado). 
CERELLA, J. Information processing rates in the elderly. Psychological Bolletin, Washington, v.98, n.1, p.67-83, 1985. CHRISTENSEN, C.L.; PAYNE, V.G.; WUGHALTER, E.H.; YAN, J.H.; HENEHAN, M.; JONES, R. Physical activity, physiological, and psychomotor performance: A study of variously active older adult men. Research Quarterly for Exercise and Sport, Washington, v.74, n.2, p.136-42, 2003.

COLCOMBE, S.; KRAMER, A. F. Fitness effects on the cognitive function of older adults: a meta-analytic study. Psychological Science, New York, v.14, n.2, 2003.

CORRÊA, U.C.; TANI, G. Aparelho de timing coincidente em tarefas complexas. P.I. n. 0,403,433-4 de 03/08/2004. Revista de Propriedade Industrial: RPI, São Paulo, n.1763, p.178, 19 out. 2004.

ERICSSON, K.A.; KRAMPE, R.T.; TESCH-RÖMER, C. The role of deliberate practice in the acquisition of expert performance. Psychological Review, Princeton, v.100, n.3, p.363-406, 1993.

FLEURY, M.; BARDY, C. Age stimulus velocity and task complexity as determiners of coincident timing behavior. Journal of Human Movement Studies, London, v.11, p.305-17, 1985.

GUIMARÃES, J.M.N; FARINATTI, P.T.V. Análise descritiva de variáveis teoricamente associadas ao risco de quedas em mulheres idosas. Revista Brasileira de Medicina do Esporte, São Paulo, v.11, n.5, p.229-305, 2005

KRAMPE, R.T.; ERICSSON, K.A. Maintaining excellence: deliberate practice and elite performance in young and older pianists. Journal of Experimental Psychology: Human Perception and Performance, Washington, v.125, n.4, p.331-59, 1996. KRAUSE, M.P.; BUZZACHERA, C.F.; HALLAGE, T.; PULNER, S.B.; SILVA, S.G. Influência do nível de atividade física sobre a aptidão cardiorrespiratória em mulheres idosas. Revista Brasileira de Medicina do Esporte, São Paulo, v.13, n.2, p.97-102, 2007. LAWTON, M.P.; BRODY, E.M. Assessment of older people: self-maintaining and instrumental activities of daily living. Gerontologist, St. Louis, v.9, n.3, p.179-86, 1969.

LOBJOIS, R.; BENGUIGUI, N.; BERTSCH, J. The effect of aging and tennis playing on coincidence: timing accuracy. Journal of Aging and Psysical Activity, Champaign, v.14, p.74-97, 2006.

MAGILL, R.A. Aprendizagem motora: conceitos e aplicações. 5. ed. São Paulo: Edgar Blucher, 2002.

MAZZEO, R.S.; CAVANAGH, P.; EVANS, W.; FIATARONE, M.; HAGBERG, J.; McAUlEY, E.; STARTZELL, J. ACSM position stand: exercise and physical activity for older adults. Medicine \& Science in Sports \& Exercise, Madison, v.30, n.6, p.992-1008, 1998.

MEEUWSEN, H.J.; GOODE, S.L.; GOGGIN, N.L. Effects of aging on coincidence anticipation timing in females. Journal of Aging and Physical Activity, Champaign, v.5, p.285-97, 1997.

NUNES, M.E.S.; SANTOS, S. Avaliação funcional de idosos em três programas de atividade física: caminhada, hidroginástica e Lian Gong. Revista Portuguesa de Ciência do Desporto, Porto, v.9, p.2-3, p.150-9, 2009.

PAROLI, R.; TANI, G. Efeitos das combinações da prática constante e variada na aquisição de uma habilidade motora. Revista Brasileira de Educação Física e Esporte, São Paulo, v.23, n.3, p.221-34, 2009.

PINHEIRO, J.P.; CORRÊA, U.C. Desempenho em uma tarefa complexa de "timing” coincidente com desaceleração do estímulo visual em indivíduos de diferentes idades. Revista Brasileira de Educação Física e Esporte, São Paulo, v.19, n.1, p.61-70, 2005. POULTON, E.C. On prediction in skilled movements. Psychological Bolletin, Washington, v.54 n.6, p.467-8, 1957.

SANTOS, S. Habilidade motora e envelhecimento. In: TANI, G. Comportamento motor: aprendizagem e desenvolvimento. Rio de Janeiro: Guanabara Koogan, 2005. p.173-206.

SCHMIDT, R.A. Movement time as a determiner of timing accuracy. Journal of Experimental Psychology: Human Perception and Performance, Washington, v.79, n.1, p.43-7, 1969.

SCHMIDT, R.A.; LEE, T.D. Motor control and learning: a behavioral emphasis. 3. ed. Champaign: Human Kinetics, 1999. SPIRDUSO, W.W. Dimensões físicas do envelhecimento. São Paulo: Manole, 2005.

TEIXEIRA, L.A. Declínio de desempenho é especifico à tarefa. Revista Brasileira de Medicina e Esporte, São Paulo, v.12, n.6, p. 351-5, 2006.

\begin{tabular}{r|r} 
ENDEREÇO & \\
Inara Marques & \\
Universidade Estadual de Londrina & Recebido para publicação: 01/ 12/ 2011 \\
Rod. Celso Garcia Cid, PR 445/km 380 - C.P. 6001 & Revisado: 02/04/2012 \\
86051-980 - Londrina - PR - BRASIL & Aceito: 02/ 05/2012 \\
e-mail: inaramarques@hotmail.com & \\
&
\end{tabular}

